\title{
Impact of Depression on Response to Comedy: A Dynamic Facial Coding Analysis
}

\author{
Lawrence Ian Reed, Michael A. Sayette, and Jeffrey F. Cohn \\ University of Pittsburgh
}

\begin{abstract}
Individuals suffering from depression show diminished facial responses to positive stimuli. Recent cognitive research suggests that depressed individuals may appraise emotional stimuli differently than do nondepressed persons. Prior studies do not indicate whether depressed individuals respond differently when they encounter positive stimuli that are difficult to avoid. The authors investigated dynamic responses of individuals varying in both history of major depressive disorder (MDD) and current depressive symptomatology $(N=116)$ to robust positive stimuli. The Facial Action Coding System (Ekman \& Friesen, 1978) was used to measure affect-related responses to a comedy clip. Participants reporting current depressive symptomatology were more likely to evince affect-related shifts in expression following the clip than were those without current symptomatology. This effect of current symptomatology emerged even when the contrast focused only on individuals with a history of MDD. Specifically, persons with current depressive symptomatology were more likely than those without current symptomatology to control their initial smiles with negative affect-related expressions. These findings suggest that integration of emotion science and social cognition may yield important advances for understanding depression.
\end{abstract}

Keywords: depression, facial expression, facial coding, comedy

Recent years have seen increased interest in applying affective science research to the study of emotional disorders (Davidson, 2000). With respect to depression, research has examined how individuals with mood disorders respond to positive and negative stimuli (Gehricke \& Shapiro, 2000). Such interest relates to early behavioral approaches for treating depression that focused on modifying one's environment to create more rewarding experiences (i.e., opportunities to increase positive affect; Lewinsohn \& Graf, 1973). This reasoning rests on the assumption that when confronted with "positive" cues, depressed individuals will respond appropriately. This assumption may not, however, be entirely accurate.

Researchers using electromyography and facial coding techniques have examined responses to stimuli intended to elicit positive emotion among individuals suffering from depression and nondepressed controls. Typically, these studies expose participants to stimuli that have reliably elicited positive affect in healthy participants (e.g., Lang, Bradley, \& Cuthbert, 1999). Following stimulus presentation, immediate responses are recorded. These studies find that individuals suffering from depression or dysphoria are less likely than healthy controls to react to positive stimuli

Lawrence Ian Reed, Michael A. Sayette, and Jeffrey F. Cohn, Department of Psychology, University of Pittsburgh.

This research was supported in part by National Institute of Mental Health Grants P01 MH056193 and R01 MH051435. We thank Ellen Frank, Charles George, and Karen Schmidt for their helpful comments. Rachel M. Levenstein and Kasey M. Griffin provided assistance with FACS coding.

Correspondence concerning this article should be addressed to Lawrence Ian Reed, Department of Psychology, 4315 Sennott Square, University of Pittsburgh, 210 S. Bouquet Street, Pittsburgh, PA 15260. E-mail: lirst6@pitt.edu with facial expressions associated with positive emotion (Gehricke \& Shapiro, 2000; Schwartz et al., 1976; Sloan, Bradley, Dimoulas, \& Lang, 2002), suggesting a diminished ability among affected persons to experience positive emotion.

With few exceptions (Sloan et al., 2002), prior research has assessed emotional reactions to positive stimuli without investigating the development of the expression over time. In addition to the morphology or configuration of these expressions, dynamic aspects of the facial movement provide information about the strength of the emotion and whether it is genuine (Ekman, 1993). More important, a facial expression of emotion may be composed of expressions occurring in a rapid sequence, conveying an emotional experience different from what is conveyed by each separate expression in the sequence (Ekman, 1993). Regarding depression, an example might be a smile control. According to Keltner (1995), smile controls involve facial actions that potentially counteract the upward pull of the smile and/or obscure the smile. Accordingly, researchers have begun to investigate these dynamic aspects of emotional expression. Analysis of motion when viewing facial expressions, for example, improves affect judgments. Specifically, subtle facial expressions were identifiable when presented in dynamic displays, but not in static displays (Ambadar, Schooler, \& Cohn, 2005). This research dovetails with prior work revealing that displays of embarrassment and amusement can be distinguished by examining temporal differences in their configurations (Keltner \& Buswell, 1997).

This basic research, which highlights the importance of dynamic measures of facial expression, may prove useful for advancing knowledge regarding mechanisms underlying depression. As noted above, clinical investigations document that persons with depression or dysphoria, on average, are less likely than nondepressed individuals to express positive affect when presented with 
positive-mood inductions. Nevertheless, this mean difference does not eliminate the possibility that some depressed participants within the group still might display positive emotional reactions. It is possible that in addition to a diminished ability to experience positive affect, there also exists an active suppression of positive affect in individuals suffering from depression. A primary aim of the present study was to examine how individuals with a history of depression (specifically major depressive disorder; MDD) who either were or were not currently suffering from depressive symptomatology respond to positive stimuli when positive responses are difficult to avoid.

Although not without debate (cf. Block \& Colvin, 1994; Taylor \& Brown, 1994), there is some evidence that individuals with depression differ from those without depression in the way they process self-relevant information. Nondepressed individuals appear to hold self-serving biases that buffer their perceptions and appraisals in ways that enhance their self-concept (Taylor \& Brown, 1988). Moreover, when emotion information that is incongruent with their self-concept cannot be avoided, there may be ways that this information is marginalized (Taylor \& Brown, 1988). In contrast, people suffering from depression may process information about the self more negatively than do their nondepressed counterparts (Abramson \& Alloy, 1981; Golin, Terrell, Weitz, \& Drost, 1979). These studies suggest that depression may alter the way that positive information is appraised, even when it cannot be avoided.

Consistent with this position, recent work using a dot-probe task suggests that individuals experiencing depression have difficulty disengaging from negative stimuli. Gotlib, Krasnoperova, Yue, and Joorman (2004) investigated attentional biases for sad faces presented for varying lengths of time. Results suggested that differences in stimulus duration may distinguish between initial orientation and maintenance of responses. Their findings further suggest that measuring affective responses dynamically will permit examination of subtle shifts in affect over brief time intervals that may characterize the reactions of individuals suffering from depression.

In order to capture such momentary shifts in emotion, we used an observational coding system to identify facial expressions thought to be related to emotion (see Ekman \& Rosenberg, 2005). The most comprehensive of these coding systems is the Facial Action Coding System (FACS; Cohn \& Ekman, 2005). FACS is an anatomically based system for measuring facial movement. Using FACS, coders can code all possible facial displays-referred to as action units (AUs) (Ekman \& Friesen, 1978; Ekman, Friesen, \& Hager, 2002)_in order to provide an objective and reliable method of measuring facial behavior over extremely rapid time frames. Thus, use of FACS to examine responses to emotion cues can provide key information that otherwise could go unnoticed if relying exclusively on self-report measures. It is hypothesized that these momentary shifts will be expressed in the face by initial positive affect-related expressions, immediately followed by expressions of negative emotion in dysphoric individuals.

Previous studies also have used affect inductions that tended not to reliably induce positive affect in depressed individuals. For example, Gehricke and Shapiro (2000) reported mean happiness ratings of less than 3 on a 0-9 scale. (More important, these low ratings were consistent with the aim of that study, which was not designed to examine expressions immediately following an initial positive response.) In contrast, the present study required a stimulus that would be especially effective in eliciting an immediate positive response, even among persons experiencing symptoms of depression. Prior studies examining the effect of depression on positive affect have not been able to determine whether difficulty responding to positive stimuli reflects a stable characteristic of individuals with depression, or instead signals current depressive symptomatology. By including groups of individuals with (a) a history of MDD and current depressive symptomatology, (b) a history of MDD without current depressive symptomatology, and (c) no history of MDD (or other psychopathology) and no current depressive symptomatology, we aimed to test the importance of current depressive symptomatology versus general vulnerability to MDD in responding to positive stimuli. Such an effort required a sample that included individuals with a history of MDD who either were currently symptomatic or currently asymptomatic.

In summary, this study tested the impact of a history of MDD, as well as current depressive symptomatology, on emotional responses to a stimulus designed to induce positive emotion. By using a robust positive stimulus, a sensitive and dynamic measure of facial behavior, and by recruiting relatively large samples of individuals with a history of MDD who were and who were not currently symptomatic, this study aimed to observe a more complex and dynamic pattern of emotion responding than found in prior studies. We hypothesized that participants with a history of MDD and currently symptomatic would be more likely to control their smiles with particular negative expressions after hearing the punch line of a comedy clip than would currently asymptomatic individuals.

\section{Method}

\section{Participants}

Participants who enrolled prior to May 2003 in a longitudinal, multidisciplinary program project examining risk factors for childhood-onset mood disorders (see Miller et al., 2002) were eligible for the present study. The present study focused on a subset $(67.1 \% ; n=116: 30$ men, 86 women) of this sample who smiled in response to a comedy clip (see the Procedures section for additional details). Included in the sample were individuals with a history of MDD and individuals without a history of psychopathology. Diagnostic information concerning history of depression was obtained via the Structured Clinical Interview for DSM-IV Patient Version (First, Spitzer, Gibbon, \& Williams, 1994), adapted to include childhood diagnoses. To be classified with a history of MDD, individuals had to receive a diagnosis of this disorder using Diagnostic and Statistical Manual of Mental Disorders, 3rd edition (DSM-III; American Psychiatric Association, 1980), the 3rd edition revised (DSM-III-R; American Psychiatric Association, 1987), or the 4th edition (DSM-IV; American Psychiatric Association, 1994) criteria before entering the study. Current depressive symptomatology was ascertained prior to film clip viewing (described in the Procedures section) using the Beck Depression Inventory (BDI; Beck, Steer, \& Farbin, 1988). Following guidelines for the BDI cut-off scores, distributed by The Center for Cognitive Therapy, current depressive symptomatology was defined as a BDI score $>18$, which corresponds to a moderate to severe level of depressive symptomatology (Beck et al., 1988). 
More details on the recruitment and diagnosis determination procedure are found in Miller et al. (2002).

To examine the effects of history of MDD and current symptomatology, participants were divided into three groups: (a) those with a history of MDD who were currently symptomatic (history + current; $n=18$ ), (b) those with a history of MDD who were not currently symptomatic (history no current; $n=39$ ), and (c) those without a history of psychopathology who were not currently symptomatic (no history no current; $n=59$ ). (Because there was only one individual in the project with elevated BDI scores but without a history of MDD, this fourth group was omitted.) The distribution of gender in each group was similar (74\% female, $26 \%$ male), as was ethnicity ( $80 \%$ Caucasian, $16 \%$ African American, $4 \%$ "other"; $p \mathrm{~s}>.10)$. There were no group differences regarding gender or race $(p s>10)$. Significant group differences were found in age, $F(2,113)=4.36, p=.015$ (see Table 1). Age, therefore, was included as a covariate in data analyses.

\section{Procedures}

Participants were seated comfortably in a chair and asked to make ratings on a series of film clips varying in emotional content. Each participant then independently viewed a neutral film clip (showing a train moving down a track), followed by a comedy clip, and then followed by five other clips that are not included in this study. The comedy clip was of the contemporary comedian Chris Rock, entitled "Chris Rock: Bring the Pain," selected on the basis of criteria outlined by Gross and Levenson (1995) to reliably elicit positive emotion. In this segment, Rock discusses a range of topics such as food and relationships. We used the first $11 \mathrm{~s}$ of this 4-min clip, which builds up to a clear, initial punch line. This resulted in a stimulus that is well suited for assessing responses, as participants generally are in a neutral state at the start of the 11-s segment. Because responses to this segment of the comedy clip are the focus of this article, the remaining film clips are not described. Video recordings of the participants' facial behavior were digitized for $11 \mathrm{~s}$ at 30 frames per second, which produced a set of 330 sequential $640 \times 480$ pixel full color images for each participant. The 11-s segment digitized for each participant began precisely $1 \mathrm{~s}$ before the punch line and ended $10 \mathrm{~s}$ after the punch line. The length of this digitized sequence allowed for analysis of spontaneous smiles that are similar in length to the average 4-6 s reported for spontaneous smiles (Frank, Ekman, \& Friesen, 1993).

FACS coding. The FACS (Ekman et al., 2002) was used to measure facial behavior. To become a certified FACS coder, one must complete a standardized exam and attain an agreement ratio of at least .70 with criteria. This reliability has been shown to generalize to research settings in which spontaneous emotion is assessed (Sayette, Cohn, Wertz, Perrott, \& Parrott, 2001).

FACS coding for the specified 11-s segment for smiles (as defined by movement of the zygomatic major muscle: AU 12) was completed by Lawrence Ian Reed (blind to history and symptomatology of participants). A number of different lower facial AUs appearing following smile onsets have been identified as reflecting smile controls (see Keltner, 1995). As noted by others (Ekman, Friesen, \& O'Sullivan, 1988), these AUs putatively modify the meaning of the smile expression, such that the overall experience may shift from reflecting joy to something different (Keltner \& Buswell, 1997). Smile controls are often low-frequency expressions, and for the purpose of this study, we examined candidate AUs that were present at least 10 times. These included movements corresponding to contempt ("dimplers" AU 14) and sadness ("lip corner depressors" AU 15; see Figure 1). Thus, the presence of a smile control (the appearance of either AU14 or AU15 during the smile) was dichotomously coded for each participant. Following Keltner (1995), these expressions were coded immediately following smile onset. Smile controls were only coded if they occurred before smile offset (following Ekman et al., 1988). Twenty percent of the participants were independently coded by a comparison coder certified in FACS and quantified using kappa, which corrects for chance agreement. Reliability for coding of smile controls was acceptable $(\kappa=.73)$.

Self-reported affect. Following both the neutral and happy film clips, self-reported happiness ratings were recorded for each participant on a 9-point Likert-type scale. (Although less relevant to the present study's aims, four other emotion terms-sadness, anger, disgust, and fear ratings - were included in the project.)

Table 1

Age, Self-Report, and Behavioral Findings by Group

\begin{tabular}{|c|c|c|c|c|c|c|}
\hline \multirow[b]{3}{*}{ Group } & \multirow{2}{*}{\multicolumn{2}{|c|}{$\begin{array}{c}\text { No history no } \\
\text { current } \\
(n=59)\end{array}$}} & \multicolumn{4}{|c|}{ History } \\
\hline & & & \multicolumn{2}{|c|}{ No current $(n=39)$} & \multicolumn{2}{|c|}{ Current $(n=18)$} \\
\hline & $M$ & $S D$ & $M$ & $S D$ & $M$ & $S D$ \\
\hline Age & $26.63^{\mathrm{a}}$ & 5.39 & $24.08^{\mathrm{b}}$ & 3.48 & $24.00^{\mathrm{a}, \mathrm{b}}$ & 4.51 \\
\hline \multicolumn{7}{|l|}{ Self-report } \\
\hline BDI & $2.63^{\mathrm{a}}$ & 3.09 & $7.67^{\mathrm{b}}$ & 5.16 & $27.11^{\mathrm{c}}$ & 7.64 \\
\hline \multicolumn{7}{|l|}{ Happiness rating } \\
\hline Neutral clip & $2.95^{\mathrm{a}}$ & 2.07 & $1.68^{\mathrm{b}}$ & 1.83 & $1.41^{\mathrm{b}}$ & 1.50 \\
\hline Comedy clip & $6.00^{\mathrm{a}}$ & 1.63 & $5.44^{\mathrm{a}}$ & 2.02 & $4.76^{\mathrm{a}}$ & 2.31 \\
\hline \multicolumn{7}{|l|}{ Behavioral } \\
\hline Smile control & $10 \%^{\mathrm{a}}$ & & $15 \%^{\mathrm{a}}$ & & $50 \%^{\mathrm{b}}$ & \\
\hline
\end{tabular}

Note. Current symptoms defined as a Beck Depression Inventory (BDI) score $>18$. Due to technical reasons or omission by the participant, happiness ratings were missing for 3 participants for the neutral clip and 5 for the comedy clip. The Group $\times$ Clip interaction regarding happiness ratings was not significant, $p=.50, F(2$, $105)=0.71$. Means with nonoverlapping superscripts differ significantly at $p<.05$ by Bonferroni correction. 


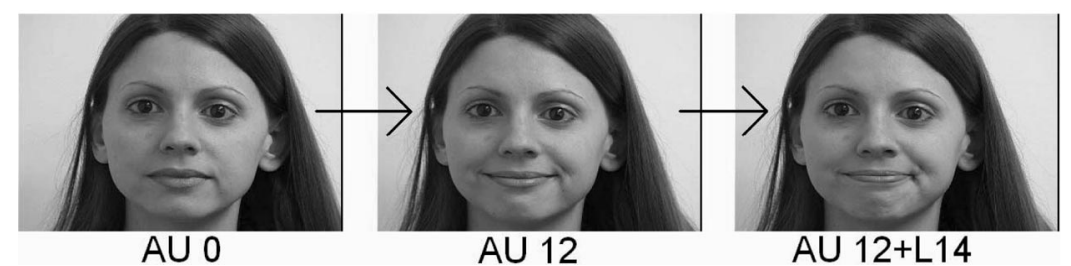

Figure 1. Example of sequence from neutral (action unit [AU] 0) to smile (AU 12) to smile control (AU $12+$ unilateral AU 14).

Results

\section{Positive Affect Induction}

We first examined whether the comedy clip was effective in eliciting positive reactions (i.e., the occurrence of smiles) across all three groups of participants. The majority (67.1\%) of participants across the three groups expressed smiles during the clip. This percentage was similar among the three groups $(58.1 \%$ history current, $66.1 \%$ history no current, and $71.1 \%$ no history no current), $\chi^{2}(2, N=173)=1.77, p=.41$.

Self-reported happiness ratings reinforced the FACS findings. [Due to technical reasons or omission by the participant, happiness ratings were missing for 3 participants for the neutral clip and 5 for the comedy clip.] More specifically, a 3 (group) $\times 2$ (rating following neutral vs. following comedy clip) mixed ANOVA revealed a main effect for clip, such that participants in all three groups reported significantly more happiness following the comedy clip, $F(1,105)=136.18, p<.001$. A main effect of group also was observed, with participants in the no-history-no-current group reporting being happiest, $F(2,105)=9.61, p<.001$. There was no Group $\times$ Clip interaction (see Table 1). The effect of watching the comedy clip on happiness was comparable in each group. Endorsement of negative emotion during the neutral and comedy clips was extremely low. On a $0-8$ Likert-type scale, all but 2 of the 24 mean scores were less than 1.0; the highest was only 1.18 .

In summary, FACS coding and self-report measures both suggested that the comedy clip was successful in providing a reliable manipulation of positive affect across the three groups.

\section{Shifts in Affect-Related Expressions}

Using age as a covariate, a logistic regression model contrasting the likelihood of observing a smile control in the three groups revealed a significant effect for group, $\chi^{2}(2, N=116)=11.71$, $p=.003$. The effect of age was not significant, $\chi^{2}(1, N=116)=$ $0.13, p=.72$.

By comparing the history-no-current group with the no-historyno-current group, we were able to contrast the effects of history of MDD among those not currently symptomatic. Results indicated that history-no-current individuals were no more likely to evince a smile control than no-history-no-current participants $(\beta=-0.42$, $p=.51)$.

By comparing the history + current group with the history-nocurrent group, we were able to contrast the effects of current symptomatology among individuals with a history of MDD. history + current in comparison with history no current were more likely to use smile controls ( $\beta=1.71, p=.009$ ). The corresponding odds ratio was 5.50 , which suggests that history + current participants were 5.5 times more likely to show a smile control.

Point biserial correlations revealed that smile controls were related to the BDI $(r=.32, p<.001)$ as well as to self-report ratings of sadness $(r=.23, p=.018)$ and disgust $(r=.21, p=$ $.025)$ following the comedy clip. Smile controls were unrelated to self-report ratings of positive affect $(r=-.14, p=.16)$ following the comedy clip.

\section{Discussion}

The major finding of this study was that among individuals with a history of MDD, those with current depressive symptomatology were more likely than were asymptomatic individuals to express smile controls during smiles. More specifically, individuals with both a history of MDD and current depressive symptomatology were more likely to express smile controls during smiles than were individuals with a history of MDD without current depressive symptomatology.

To our knowledge, this is the first study using FACS to measure dynamic affect-related shifts in a sample with a history of MDD and current depressive symptomatology. These data reinforce prior studies that found that individuals suffering from depression responded to positive stimuli with diminished responses. Our study found that even when an initial smile is elicited, individuals with current symptomatology are more likely than asymptomatic persons to express negative AUs that are thought to control their original positive response (Ekman et al., 1988). Although prior work has not been able to disentangle the impact of a history of MDD from an individual's current state (as patients typically were recruited on the basis of current diagnosis), the present study provides preliminary evidences that it is the current state, rather than some stable tendency related to a history of MDD, that is driving the response to positive stimuli.

The momentary shifts in expression captured by FACS were not observed in our postcomedy clip affect ratings. Nor did we find group differences in initial positive facial responses. The similar initial responses to the joke may have been due to the effectiveness of our comedy clip in eliciting positive responses. Positive affective ratings in the present study were higher than those found in previous research using film clips (e.g., Rottenberg, Kasch, Gross, \& Gotlib, 2002). Although we believe that this difference is due to the effectiveness of our comedy clip, it remains possible that it is a result of the way in which depression is defined in the present study. Increases in positive affect ratings following the comedy 
clip did not differ by group, suggesting similar response patterns between groups.

The FACS data reported here are consistent with the view that depressive symptomatology can bias one toward negative interpretations of cues. It also provides a complement to work suggesting that currently depressed individuals have difficulty disengaging from negative stimuli (Gotlib et al., 2004) and work suggesting that depression severity might be associated with aversive responses to positive stimuli (Allen, Trinder, \& Brennan, 1999). Our data also are consistent with views of depression that consider negative processing biases to be the result of well-learned routines, or heuristics (Robinson, Goetz, Wilkowski, \& Hoffman, 2006). Alternatively, participants expressing smile controls simply may have been responding with ambivalence. Future research to probe these different sources of bias is indicated.

Together with other research (e.g., Allen et al., 1999; Gotlib et al., 2004), our findings suggest that, under certain conditions, individuals with current depressive symptomatology may actively process information in order to create affectively congruent responses. This position differs from traditional conceptualizations that link depression to a passive style of responding (Beck, 1967). Accordingly, merely encouraging such patients to surround themselves with positive stimuli or situations may not be sufficient in treatment if attention is not also paid to how these stimuli might be appraised. Behavioral treatments for depression that involve behavioral activation recognize that individuals may not all find the same stimuli reinforcing (Gable, Reis, \& Elliot, 2000). With respect to laboratory research, use of idiographic approaches to assessing positive affect may prove valuable.

Given the important communicative function of facial expression (Fridlund, 1991), it is possible that the display of smile controls by depressed individuals may alienate them from others. This possibility may provide a clue toward understanding how the behavior of depressed people may elicit hostility and rejection from others (see Coyne, 1990).

A number of limitations to this study must be taken into account in interpreting its findings. Because most of the participants included in the sample were in their 20s, it is possible that some of those classified without a history might develop MDD later in adulthood. Regarding our sample, a no history + current group was not included in the analyses due to its small sample size. Thus, we were unable to contrast the effects of current symptomatology among individuals with a history of MDD by comparing a no history + current group with the history + current group. Moreover, the majority of participants in the history-no-current $(77 \%)$ and history + current $(78 \%)$ groups were diagnosed with MDD prior to age 16 . Because early onset depression is associated with poor adult outcome compared with later onset depression (e.g., Lewinsohn, Clarke, Seeley, \& Rohde, 1994), future research is needed to determine how well our findings generalize to individuals with later onset depression.

In addition, use of a self-reported measure of depressive symptomatology can be problematic. First, rather than strictly measuring depressive symptomatology, the BDI may represent a more global measure of negative affect. Second, any BDI cut-off score is arbitrary. Thus, results could change with the use of different cut-off scores. It also is possible that the BDI cut-off scores function differently in those with and without a history of MDD. More generally, it remains unclear whether the observed pattern of smile controls would be maintained in response to other types of emotion inductions (e.g., unpleasant and neutral stimuli).

The present data suggest that affective reactions in individuals with a history of MDD with and without current symptomatology are more likely to unfold differently over time than in asymptomatic "healthy" persons. Future research using other techniques (e.g., brain imaging) may provide further support for the conclusion that depression is associated with a complex controlling response to positive information.

This research represents an initial attempt to use a dynamic measure of affect-related expression to examine the unfolding of emotion in individuals with depressive symptomatology. More generally, this study highlights the utility of integrating basic research in emotion with the study of affective disorders. Such research holds promise for improving understanding of mechanisms underlying depression, while also providing a fertile sample for investigating emotional volatility. Microanalysis of such rapidly changing affect-related expressions may yield new insights into theories of human emotion.

\section{References}

Abramson, L. Y., \& Alloy, L. B. (1981). Depression, non-depression, and cognitive illusions: A reply to Schwartz. Journal of Experimental Psychology, 110, 436-447.

Allen, N. B., Trinder, J., \& Brennan, C. (1999). Affective startle modulation in clinical depression: Preliminary findings. Biological Psychiatry, $46,542-550$.

Ambadar, Z., Schooler, J. W., \& Cohn, J. F. (2005). Deciphering the enigmatic face: The importance of facial dynamics in interpreting subtle facial expressions. Psychological Science, 16, 403-410.

American Psychiatric Association. (1980). Diagnostic and statistical manual of mental disorders (3rd ed.). Washington, DC: Author.

American Psychiatric Association. (1987). Diagnostic and statistical manual of mental disorders (3rd ed., rev.). Washington, DC: Author.

American Psychiatric Association. (1994). Diagnostic and statistical manual of mental disorders (4th ed.). Washington, DC: Author.

Beck, A. T. (1967). Depression: Clinical, experimental, and theoretical aspects. New York: Hoeber.

Beck, A. T., Steer, R. A., \& Farbin, M. G. (1988). Psychometric properties of the Beck Depression Inventory: Twenty-five years of evaluation. Clinical Psychology Review, 8, 77-100.

Block, J., \& Colvin, C. R. (1994). Positive illusions and well-being revisited: Separating fiction from fact. Psychological Bulletin, 116, 28.

Cohn, J. F., \& Ekman, P. (2005). Measuring facial action by manual coding, facial EMG, and automatic facial image analysis. In J. A. Harrigan, R. Rosenthal, \& K. Scherer (Eds.), Handbook of nonverbal behavior research methods in the affective sciences (pp. 9-64). New York: Oxford University Press.

Coyne, J. C. (1990). Interpersonal processes in depression. In G. L. Keitner (Ed.), Depression and families (pp. 31-54). Washington, DC: American Psychiatric Press.

Davidson, R. J. (2000). Anxiety, depression, and emotion. New York: Oxford University Press.

Ekman, P. (1993). Facial expression and emotion. American Psychologist, 48, 384-392.

Ekman, P., \& Friesen, W. V. (1978). Facial Action Coding System. Palo Alto, CA: Consulting Psychology Press.

Ekman, P., Friesen, W. V., \& Hager, J. C. (2002). The Facial Action Coding System. Salt Lake City, UT: Research Nexus, Network Research Information.

Ekman, P., Friesen, W. V., \& O’Sullivan, M. (1988). Smiles when lying. Journal of Personality and Social Psychology, 54, 414-420. 
Ekman, P., \& Rosenberg, E. (2005). What the face reveals: Basic and applied studies of spontaneous expression using the facial action coding system (FACS). New York: Oxford University Press.

First, M. B., Spitzer, R. L., Gibbon, M., \& Williams, J. B. W. (1994). Structured clinical interview for axis I DSM-IV disorders. SCID-I/ $P(2.00)$. New York: Biometrics Research Department.

Frank, M. G., Ekman, P., \& Friesen, W. V. (1993). Behavioral markers and recognizability of the smile of enjoyment. Journal of Personality and Social Psychology, 64, 83-93.

Fridlund, A. J. (1991). Sociality of solitary smiling: Potentiation by an implicit audience. Journal of Personality and Social Psychology, 60, 229-240.

Gable, S. L., Reis, H. T., \& Elliot, A. (2000). Behavioral activation and inhibition in everyday life. Journal of Personality and Social Psychology, 78, 1135-1149.

Gehricke, J. G., \& Shapiro, D. (2000). Reduced facial expression and social context in major depression: Discrepancies between facial muscle activity and self-reported emotion. Psychiatry Research, 95, 157-167.

Golin, S., Terrell, T., Weitz, J., \& Drost, P. L. (1979). The illusion of control among depressed patients. Journal of Abnormal Psychology, 88, $454-457$.

Gotlib, I. H., Krasnoperova, E., Yue, D. N., \& Joorman, J. (2004). Attentional biases for negative interpersonal stimuli in clinical depression. Journal of Abnormal Psychology, 113, 127-135.

Gross, J., \& Levenson, R. W. (1995). Emotion elicitation using films. Cognition \& Emotion, 9, 81-108.

Keltner, D. (1995). Signs of appeasement: Evidence for the distinct displays of embarrassment, amusement, and shame. Journal of Personality and Social Psychology, 68, 441-454.

Keltner, D., \& Buswell, B. N. (1997). Embarrassment: Its distinct form and appeasement functions. Psychological Bulletin, 122, 250-270.

Lang, P. J., Bradley, M. M., \& Cuthbert, B. M. (1999). International affective picture system (IAPS): Instructional manual and affective ratings. Gainesville: University of Florida, Center for Research in Psychophysiology.

Lewinsohn, P. M., Clarke, G. N., Seeley, J. R., \& Rohde, P. (1994). Major depression in community adolescents: Age at onset, episode duration, and time to recurrence. Journal of the American Academy of Child \& Adolescent Psychiatry, 33, 809-818.

Lewinsohn, P. M., \& Graf, M. (1973). Pleasant activities and depression. Journal of Consulting and Clinical Psychology, 41, 261-268.

Miller, A., Fox, N. A., Cohn, J. F., Forbes, E. E., Sherrill, J. T., \& Kovacs, M. (2002). Regional patterns of brain activity in adults with a history of childhood-onset depression: Gender differences and clinical variability. American Journal of Psychiatry, 159, 934-940.

Robinson, M. D., Goetz, M. C., Wilkowski, B. M., \& Hoffman, S. J. (2006). Driven to tears or to joy: Response dominance and trait-based predictions. Personality and Social Psychology Bulletin, 32, 629-640.

Rottenberg, J., Kasch, K. L., Gross, J. J., \& Gotlib, I. H. (2002). Sadness and amusement reactivity differentially predict concurrent and prospective functioning in major depressive disorder. Emotion, 2, 135-146.

Sayette, M. A., Cohn, J. F., Wertz, J. M., Perrott, M. A., \& Parrott, D. J. (2001). A psychometric evaluation of the Facial Action Coding System for assessing spontaneous expression. Journal of Nonverbal Behavior, $25,167-185$.

Schwartz, G. E., Fair, P. L., Mandel, M. R., Salt, P., Mieske, M., \& Klermen, G. L. (1976, April 30). Facial muscle patterning to affective imagery in depressed and nondepressed subjects. Science, 192, 489491.

Sloan, D. M., Bradley, M. M., Dimoulas, E., \& Lang, P. J. (2002). Looking at facial expressions: Dysphoria and facial EMG. Biological Psychology, 60, 79-90.

Taylor, S. E., \& Brown, J. D. (1988). Illusion and well-being: A social psychological perspective on mental health. Psychological Bulletin, 102, 193-210.

Taylor, S. E., \& Brown, J. D. (1994). Positive illusions and well-being revisited: Separating fact from fiction. Psychological Bulletin, 116, 21-27.

Received May 16, 2006 Revision received April 13, 2007 Accepted April 16, 2007
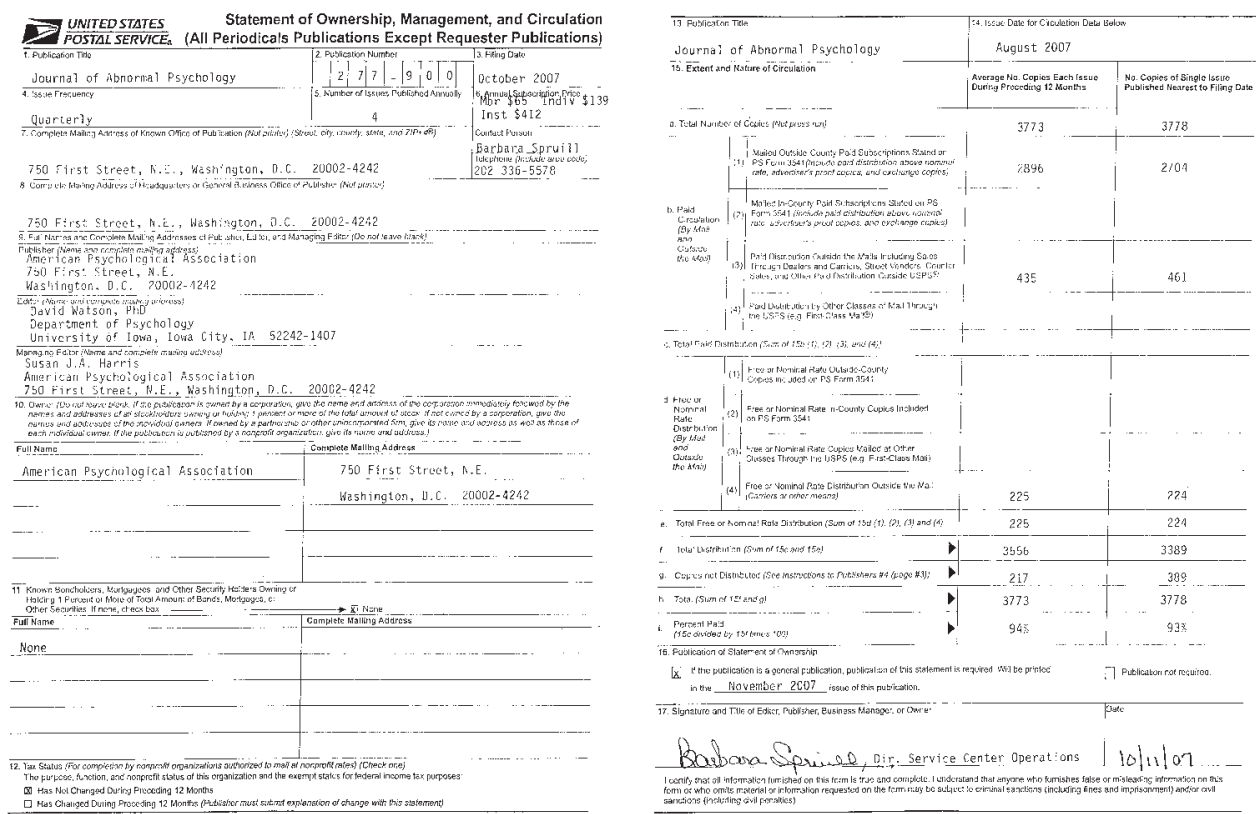